\title{
SPECTRA OF THE DIFFERENCE AND PRODUCT OF PROJECTIONS
}

\author{
MATJAŽ OMLADIČ
}

\begin{abstract}
A short natural proof of the known relation between spectra of the difference and the product of two orthogonal projections is given. This proof also generalizes this result.
\end{abstract}

The problem of how to determine the spectrum of the product of two orthogonal projections in an arbitrary complex Hilbert space $H$ from the spectrum of their difference arises from statistics and was recently solved for point spectrum by W. N. Anderson, Jr., E. J. Harner, and G. E. Trapp [1]. Here is their result, stated for the whole spectrum.

THEOREM. For any pair of orthogonal projections $P$ and $Q$ on $H$ the spectrum of the product $P Q$ lies in the interval $[0,1]$ and

$$
\sigma(Q-P) \backslash\{-1,0,1\}=\left\{ \pm(1-\mu)^{1 / 2} ; \mu \in \sigma(P Q) \backslash\{0,1\}\right\} .
$$

REMARK. Note that this Theorem can be equivalently formulated as follows: For any pair of orthogonal projections $P$ and $Q$ on $H$ the spectrum of $Q-P$ lies in the interval $[-1,1], \sigma(Q-P) \backslash\{-1,0,1\}=\sigma(P-Q) \backslash\{-1,0,1\}$, and

$$
\sigma(P Q) \backslash\{0,1\}=\left\{1-\lambda^{2} ; \lambda \in \sigma(Q-P) \backslash\{-1,0,1\}\right\} .
$$

Proof. Write $U=\operatorname{Im} P, V=\operatorname{Ker} P$, decompose the space $H$ into $H=U \oplus V$, and represent the operator $Q$ as an operator matrix (see [2] for definition of operator matrices)

$$
Q=\left[\begin{array}{cc}
A & B \\
B^{*} & C
\end{array}\right]
$$

Note that $A$ is a selfadjoint operator on $U, C$ is a selfadjoint operator on $V$, and that $\sigma(A) \subset[0,1]$ and $\sigma(C) \subset[0,1]$. From $Q^{2}=I$ we get

$$
B B^{*}=A-A^{2}, \quad B^{*} B=C-C^{2}, \quad \text { and } \quad A B+B C=B
$$

which implies that $\operatorname{Ker} B \subset V$ is invariant under $C$ and must therefore reduce this operator. Moreover, it is clear for the restriction $\left.C\right|_{\operatorname{Ker} B}$ that $\sigma\left(\left.C\right|_{\operatorname{Ker} B}\right) \subset\{0,1\}$. Similarly, the subspace $\operatorname{Ker} B^{*} \subset U$ reduces $A$ and $\sigma\left(\left.A\right|_{\operatorname{Ker} B^{*}}\right) \subset\{0,1\}$. Denote

$$
A_{1}=\left.A\right|_{\overline{\operatorname{Im} B}}, \quad C_{1}=\left.C\right|_{\overline{\operatorname{Im} B^{*}}}, \quad \text { and } \quad B_{1}=\left.B\right|_{\overline{\operatorname{Im} B^{*}}} ;
$$

then, $B_{1}: \overline{\operatorname{Im} B^{*}} \rightarrow \overline{\operatorname{Im} B}$ is a quasiaffinity, i.e. a bounded injective operator with dense range. In addition, $B_{1}$ intertwines $C_{1}$ and $I-A_{1}$, i.e. $B_{1} C_{1}=\left(I-A_{1}\right) B_{1}$. It is well known that this forces the spectral measures $E$ of $C_{1}$ and $F$ of $I-A_{1}$ to satisfy

Received by the editors December 30, 1985.

1980 Mathematics Subject Classification (1985 Revision). Primary 47A10.

This work was supported by the Research Council of Slovenia. 
the relation $B_{1} E(\sigma)=F(\sigma) B_{1}$ for any interval $\sigma$ of the real line. Consequently, the projections $E(\sigma)$ and $F(\sigma)$ are zero together which yields $\sigma\left(C_{1}\right)=\sigma\left(I-A_{1}\right)$. It follows that $\sigma(C) \backslash\{0,1\}=\sigma(I-A) \backslash\{0,1\}$.

The form which the matrix $(\lambda-Q+P)^{-1}$ takes in the 2-dimensional case, where

$$
P=\left[\begin{array}{ll}
1 & 0 \\
0 & 0
\end{array}\right] \text { and } Q=\left[\begin{array}{ll}
\cos ^{2} \vartheta & \sin \vartheta \cos \vartheta \\
\sin \vartheta \cos \vartheta & \sin ^{2} \vartheta
\end{array}\right] \text {, }
$$

suggests our defining the following operator matrix $R(\lambda)$ for every complex $\lambda$ for which it makes sense:

$$
R(\lambda)=\left[\begin{array}{ll}
I+\left(\lambda-\lambda^{2}\right)\left(A-I+\lambda^{2}\right)^{-1} & B\left(\lambda^{2}-C\right)^{-1} \\
B^{*}\left(A-I+\lambda^{2}\right)^{-1} & -I+\left(\lambda+\lambda^{2}\right)\left(\lambda^{2}-C\right)^{-1}
\end{array}\right] .
$$

Use the above relations between the operators $A, B$, and $C$ to verify

$$
(\lambda-Q+P) R(\lambda)=R(\lambda)(\lambda-Q+P)=I .
$$

Representing also the operator $P Q$ by the operator matrix

$$
P Q=\left[\begin{array}{cc}
A & B \\
0 & 0
\end{array}\right]
$$

we see that $\sigma(P Q) \backslash\{0\}=\sigma(A) \backslash\{0\}$. Take any $\mu \in \rho(P Q), \mu \notin\{0,1\}$, to see that $\mu \in \rho(A)$ and $1-\mu \in \rho(C)$. Put either $\lambda=(1-\mu)^{1 / 2}$, or $\lambda=-(1-\mu)^{1 / 2}$ into $R(\lambda)$ to get that both belong to $\rho(Q-P)$. To obtain the inverse inclusion, take any $\lambda \in \rho(Q-P), \lambda \notin\{-1,0,1\}$, and note that $R(\mu)$ converges to $(\lambda-P+Q)^{-1}$ when $\mu$ approaches the point $\lambda$ through complex values. It follows that $\left(A-I+\lambda^{2}\right)^{-1}$ exists and that

$$
\left(A-I+\lambda^{2}\right)^{-1}=\left(R(\lambda)^{P}-I\right) /\left(\lambda-\lambda^{2}\right),
$$

where $R(\lambda)^{P}$ denotes the compression of $R(\lambda)$ to the space $U$. Therefore, $1-\lambda^{2} \in$ $\rho(A)$ and consequently $1-\lambda^{2} \in \rho(P Q)$.

ACKNOWLEDGMENT. The author would like to thank M. Hladnik for a helpful conversation and the referee for helping him improve the text.

\section{REFERENCES}

1. W. N. Anderson, Jr., E. J. Harner, and G. E. Trapp, Ergenvalues of the difference and product of projections, Linear and Multilinear Algebra 17 (1985), 295-299.

2. P. R. Halmos, A Hilbert space problem book, Springer, New York and Heidelberg, 1974.

Department of MAThematics, E. K. University of LJUbljanA, LjubljanA, YUGOSLAVIA 